\title{
A New Vector Partition of the Probability Score
}

\author{
AlLAN H. MURPHY \\ National Center for Atmospheric Research, ${ }^{1}$ Boulder, Colo. 80302
}

(Manuscript received 19 October 1972, in revised form 12 March 1973)

\begin{abstract}
A new vector partition of the probability, or Brier, score $(P S)$ is formulated and the nature and properties of this partition are described. The relationships between the terms in this partition and the terms in the original vector partition of the $P S$ are indicated. The new partition consists of three terms: 1) a measure of the uncertainty inherent in the events, or states, on the occasions of concern (namely, the $P S$ for the sample relative frequencies) ; 2) a measure of the reliability of the forecasts; and 3) a new measure of the resolution of the forecasts. These measures of reliability and resolution are and are not, respectively, equivalent (i.e., linearly related) to the measures of reliability and resolution provided by the original partition. Two sample collections of probability forecasts are used to illustrate the differences and relationships between these partitions. Finally, the two partitions are compared, with particular reference to the attributes of the forecasts with which the partitions are concerned, the interpretation of the partitions in geometric terms, and the use of the partitions as the bases for the formulation of measures to evaluate probability forecasts. The results of these comparisons indicate that the new partition offers certain advantages vis-à-vis the original partition.
\end{abstract}

\section{Introduction}

Murphy (1972a, b) has recently described scalar and vector partitions of the probability, or Brier, score $(P S)$ (Brier, 1950). These partitions, which provide similar, but not equivalent (i.e., linearly related), measures of the reliability and resolution of probability forecasts, are generalizations of a partition of the $P S$ originally formulated by Sanders $(1958,1963)$. Hereafter, we shall refer to these partitions as the original partitions of the $P S$.

The purposes of this paper are to describe a new partition of the $P S$ and to compare this partition with the original partition. In this paper we consider only vector partitions, since discussions concerning the relative merits of the original scalar and vector partitions of the $P S$ indicate that vector partitions are, in general, more appropriate than scalar partitions (see Murphy, 1972a, b).

In Section 2 we define the $P S$ and briefly describe the original vector partition of the $P S$. The new vector partition of the $P S$ is formulated and the nature and properties of this partition are described in Section 3. In Sections 4 and 5 we indicate the relationships between the new and original partitions and illustrate the differences between these partitions in terms of sample collections of forecasts, respectively. The new and original partitions are compared in Section 6, with particular reference to the attributes of the forecasts with which the partitions are concerned, the interpretation of the partitions in geometric terms, and the use of the

${ }^{1}$ The National Center for Atmospheric Research is sponsored by the National Science Foundation. partitions as the bases for the formulation of measures to evaluate probability forecasts. Section 7 consists of a brief summary and conclusion.

\section{The original vector partition of the probability score}

a. The PS

Consider a meteorological variable whose range has been divided into $N$ mutually exclusive and collectively exhaustive states $\left\{s_{1}, \ldots, s_{N}\right\}$. Let the row vector $\mathbf{r}_{k}=\left(r_{1 k}, \ldots, r_{N k}\right) \quad\left(r_{n k} \geqslant 0, \sum_{n} r_{n k}=1 ; n=1, \ldots, N\right.$; $k=1, \ldots, K)$ denote the $k$ th forecast in a collection of $K$ forecasts, where $r_{n k}$ is the forecast probability of state $s_{n}$ on the $k$ th occasion, and let the row vector $\mathbf{d}_{k}$ $=\left(d_{1 k}, \ldots, d_{N k}\right)$ denote the $k$ th observation in the collection of $K$ relevant observations, where $d_{n k}$ equals one if state $s_{n}$ obtains on the $k$ th occasion and zero otherwise. Then, the PS for the collection of $K$ forecasts $\mathbf{r}_{k}(k=1, \ldots, K)$ is $P S(\mathbf{r}, \mathbf{d})$, where

$$
P S(\mathbf{r}, \mathbf{d})=(1 / K) \sum_{k=1}^{K}\left(\mathbf{r}_{k}-\mathbf{d}_{k}\right)\left(\mathbf{r}_{k}-\mathbf{d}_{k}\right)^{\prime},
$$

in which a prime denotes a column vector. The range of $P S(\mathbf{r}, \mathbf{d})$, in $(1)$, is the closed interval $[0,2]$ and $P S(\mathbf{r}, \mathbf{d})$ has a negative orientation (i.e., the smaller the score the better the score).

\section{b. The original vector partition}

The partitions of the $P S$ of concern in this paper are based upon the assumption that the probabilities, $r_{n k}$, 
which constitute the forecasts, $\mathbf{r}_{k}$, can assume only a finite set of $S$ distinct values, $r^{s}$, i.e., $r_{n k}=r^{s}$ for all $n$ and $k$ for some $s(n=1, \ldots, N ; k=1, \ldots, K ; s=1$, $\ldots, S)$. Then, we can identify $T$ distinct vector forecasts $\mathbf{r}^{t}(t=1, \ldots, T)$, where

$$
T=\sum_{s=1}^{S}\left(\begin{array}{c}
N+s-4 \\
s-1
\end{array}\right)(S-s+1),
$$

in which $\left(\begin{array}{l}x \\ y\end{array}\right)=x ! /[y !(x-y) !]$ for $0 \leqslant y \leqslant x,\left(\begin{array}{l}x \\ y\end{array}\right)=1$ for $x=-1$ and $y=0$, and $\left(\begin{array}{l}x \\ y\end{array}\right)=0$ otherwise (see Murphy, 1972b, Footnote 5). Thus, we can identify $T$. distinct subcollections of the collection of $K$ forecasts, where subcollection $t$ consists of the $K^{t}$ forecasts for which

$$
\mathbf{r}_{k}=\mathbf{r}^{t}, \quad\left(k=1, \ldots, K^{t} ; \sum_{t} K^{t}=K ; t=1, \ldots, T\right),
$$

in which $\mathbf{r}^{t}=\left(r_{1}^{t}, \ldots, r_{N}^{t}\right)$. Now, Murphy (1972b) has shown that $P S(\mathbf{r}, \mathbf{d})$, in $(1)$, can be partitioned as follows:

$$
\begin{aligned}
P S(\mathbf{r}, \mathbf{d})=(1 / K) \sum_{t=1}^{T} K^{t}\left(\mathbf{r}^{t}-\overline{\mathrm{d}}^{t}\right)\left(\mathbf{r}^{t}-\mathrm{d}^{t}\right)^{\prime} & \\
& +(1 / K) \sum_{t=1}^{T} K^{t} \mathbf{d}^{t}(\mathbf{u}-\overline{\mathrm{d}})^{\prime},
\end{aligned}
$$

where $\overline{\mathrm{d}}^{t}=\left(\bar{d}_{1}^{t}, \ldots, \bar{d}_{N}^{t}\right)$, in which

$$
\bar{d}_{n}^{t}=\left(1 / K^{t}\right) \sum_{k=1}^{K^{\ell}} d_{n k}^{t}, \quad(n=1, \cdots, N),
$$

and $\mathbf{u}$ is the unity vector, a row vector whose $N$ elements are all equal to one [i.e., $\mathbf{u}=(1, \ldots, 1)] . P S(\mathbf{r}, \mathbf{d})$, in (2), represents the original vector partition of the $P S$.

The first term on the right-hand side (RHS) of (2) is a measure of the degree to which the $T$ distinct forecasts $\mathbf{r}^{t}$ differ from the respective sample relative frequencies $\overline{\mathbf{d}}^{t}(t=1, \ldots, T)$. The second term on the RHS of (2) is a measure of the degree to which the sample relative frequencies for the $T$ subcollections of forecasts, $\overline{\mathbf{d}}^{t}(t=1, \ldots, T)$, differ from either the null vector $\mathbf{n}=(0, \ldots, 0)$ or the unity vector $\mathbf{u}=(1, \ldots, 1)$. These two terms were referred to originally as measures of the attributes reliability and resolution, respectively (Sanders, 1958), and subsequently as measures of the attributes validity and sharpness, respectively (Sanders, 1963). We shall use the original terms because we prefer to define the term validity in a more general manner and because the term sharpness relates to the degree to which the forecast probabilities, rather than the sample relative frequencies, differ from either the null vector or the unity vector (see Murphy, 1972a, Footnote 10). On the other hand, this measure of resolution is similar in certain respects to at least one measure of sharpness (see Section 6). Finally, note that the ranges of these terms are the closed intervals $[0,2]$ and
$[0,(N-1) / N]$, respectively, and that the smaller these terms the greater the reliability and resolution, respectively, of the forecasts and the smaller the $P S$.

\section{A new vector partition of the probability score \\ a. Formulation}

The original vector partition of the $P S, P S(\mathbf{r}, \mathbf{d})$, in (2), can also be expressed as

$$
\begin{aligned}
P S(\mathbf{r}, \mathbf{d})=(1 / K) \sum_{t=1}^{T} K^{t}\left(\mathbf{r}^{t}-\mathbf{d}^{t}\right)\left(\mathbf{r}^{t}-\overline{\mathrm{d}}^{t}\right)^{\prime}+\overline{\mathrm{d}} \mathbf{u}^{\prime} & \\
& -(1 / K) \sum_{t=1}^{T} K^{t} \overline{\mathrm{d}}^{t}\left(\overline{\mathrm{d}}^{t}\right)^{\prime},
\end{aligned}
$$

where $\overline{\mathrm{d}}=\left(\bar{d}_{1}, \ldots, \bar{d}_{N}\right)$, in which

$$
\bar{d}_{n}=(1 / K) \sum_{t=1}^{T} K^{t} \bar{d}_{u}^{t}, \quad(n=1, \ldots, n)
$$

In addition, if we add and subtract the quantity $\overline{\mathrm{d}} \overline{\mathrm{d}}^{\prime}$ on the RHS of (3), then we obtain

$$
\begin{array}{r}
P S(\mathbf{r}, \mathbf{d})=(1 / K) \sum_{t=1}^{T} K^{t}\left(\mathbf{r}^{t}-\overline{\mathrm{d}}^{t}\right)\left(\mathbf{r}^{t}-\overline{\mathrm{d}}^{t}\right)^{\prime}+\overline{\mathrm{d}}(\mathbf{u}-\overline{\mathrm{d}})^{\prime} \\
-\left[\overline{\mathrm{d}} \overline{\mathrm{d}}^{\prime}-2 \overline{\mathrm{d}} \overline{\mathrm{d}}^{\prime}+(1 / K) \sum_{t=1}^{T} K^{t} \overline{\mathbf{d}}^{\prime}(\overline{\mathrm{d}})^{\prime}\right],
\end{array}
$$

ol:

$$
\begin{aligned}
F S(\mathbf{r}, \mathbf{d})=\overline{\mathrm{d}}(\mathbf{u}-\overline{\mathrm{d}})^{\prime} & +(\mathbf{1} / K) \sum_{t=1}^{T} K^{t}\left(\mathbf{r}^{t}-\overline{\mathrm{d}}^{t}\right)\left(\mathbf{r}^{t}-\overline{\mathrm{d}}^{t}\right)^{\prime} \\
& -(1 / K) \sum_{t=1}^{T} K^{t}\left(\overline{\mathrm{d}}^{t}-\overline{\mathrm{d}}\right)\left(\overline{\mathrm{d}}^{t}-\overline{\mathrm{d}}\right)^{\prime} .
\end{aligned}
$$

Now, from (1),

$$
P S(\overline{\mathrm{d}}, \mathbf{d})=\overline{\mathrm{d}}(\mathbf{u}-\overline{\mathrm{d}})^{\prime} .
$$

Thus, PS(r,d), in (4), becomes

$$
\begin{aligned}
P S(\mathbf{r}, \mathbf{d})=P S(\overline{\mathbf{d}}, \mathbf{d})+ & (1 / K) \sum_{t=1}^{T} K^{t}\left(\mathbf{r}^{t}-\overline{\mathrm{d}}^{t}\right)\left(\mathbf{r}^{t}-\overline{\mathrm{d}}^{t}\right)^{\prime} \\
& -(1 / K) \sum_{t=1}^{T} K^{t}\left(\overline{\mathrm{d}}^{t}-\overline{\mathbf{d}}\right)\left(\overline{\mathbf{d}}^{t}-\overline{\mathbf{d}}\right)^{\prime}
\end{aligned}
$$

$P S(\mathbf{r}, \mathbf{d})$, in (6), represents the new vector partition of the $P S$.

\section{b. Nature and properties}

The first term of the RHS of (6) simply represents the $P S$ that would be obtained if each forecast $\mathbf{r}_{k}(k=1, \ldots, K)$ were replaced by the sample relative frequencies for the collection of $K$ forecasts, $\overline{\mathrm{d}}$. Thus, this term is a measure of the uncertainty inherent in the events, or states, on the $K$ occasions of concern. Note that the range of this term is the closed interval $[0,(N-1) / N]$ and that the larger this term the greater 
the uncertainty and the greater the PS. The second term on the RHS of (6) is identical to the first term on the RHS of (2) and, as such, is a measure of the reliability of the collection of $K$ forecasts. Recall that the range of this term is the closed interval $[0,2]$ and that the smaller this term the greater the reliability and the smaller the PS. The third term on the RHS of (6) is a measure of the degree to which the sample relative frequencies for the $T$ subcollections of forecasts, $\overline{\mathrm{d}}^{t}(t=1, \ldots, T)$, differ from the sample relative frequencies for the collection of $K$ forecasts, $\overline{\mathrm{d}}$. This term, then, is not a measure of the same attribute of the forecasts as the second term on the RHS of (2). However, in the absence of more appropriate terminology, we shall refer to this term as a measure of the resolution of the forecasts. In this regard, the reader should bear in mind that these two measures of resolution relate to different attributes of the forecasts and that the measures themselves are not equivalent (see Sections 4 and 5). Finally, note that the range of this term is the closed interval $\left[0,(N-1) / N^{\prime}\right]$ and that the smaller this term the smaller the resolution and the greater the $P S$.

\section{The new and original partitions: The relation- ship}

What, if any, are the relationships between the terms in the new and original vector partitions of the PS? Note that, since these partitions are both partitions of the $P S$, we can equate the RHS of (2) and the RHS of (6), in which case we find that

$$
\begin{aligned}
P S(\mathbf{d}, \mathbf{d})=(1 / K) \sum_{t=1}^{T} K^{t}\left(\mathbf{d}^{t}-\mathbf{d}\right)\left(\mathrm{d}^{t}-\mathrm{d}\right)^{\prime} & \\
& +(1 / K) \sum_{t=1}^{T} K^{t} \mathrm{~d}^{t}\left(\mathbf{u}-\mathbf{d}^{t}\right)^{\prime} .
\end{aligned}
$$

That is, the measure of uncertainty, $P S(\overline{\mathbf{d}}, \mathbf{d})$, is the sum of the new and original measures of the resolution of the forecasts. Thus, the two measures of resolution relate to different aspects of the uncertainty inherent in the states on the $K$ occasions of concern, and, in
TABLE 1. A sample collection of forecasts and the relevant observations in the two-state situation.

\begin{tabular}{ccc}
$\begin{array}{c}\text { Forecast } \\
\text { Observation } \\
\text { number } \\
k\end{array}$ & $\begin{array}{c}\text { Forecast } \\
\mathbf{1}_{k}\end{array}$ & $\begin{array}{c}\text { Observation } \\
\mathbf{d}_{k}\end{array}$ \\
\hline 1 & $(0.2,0.8)$ & $(0,1)$ \\
2 & $(0.6,0.4)$ & $(1,0)$ \\
3 & $(0.9,0.1)$ & $(1,0)$ \\
4 & $(0.2,0.8)$ & $(0,1)$ \\
5 & $(0.1,0.9)$ & $(0,1)$ \\
6 & $(0.2,0.8)$ & $(0,1)$ \\
7 & $(0.4,0.6)$ & $(1,0)$ \\
8 & $(0.7,0.3)$ & $(1,0)$ \\
9 & $(0.8,0.2)$ & $(1,0)$ \\
10 & $(0.2,0.8)$ &
\end{tabular}

this regard, the measures are complementary (in a mathematical sense). We consider these measures in greater detail in Section 6.

\section{The new and original partitions: Sample collec- tions of forecasts}

In order to illustrate the new partition of the $P S$ and to compare the values of the terms in the new and original partitions of the $P S$, we consider sample collections of forecasts in the two-state $(N=2)$ and threestate $(N=3)$ situations in this section. For comparative purposes, we use the same collections of forecasts as those used to illustrate the original vector partition of the PS (see Murphy, 1972a, b).

A sample collection of ten $(K=10)$ forecasts in the two-state situation is presented in Table 1 (cf. Murphy, $1972 \mathrm{a}$, Table 1b). The new vector partition of the $P S$ for these forecasts is presented in Table 2. The sample relative frequencies for this collection of forecasts, $\overline{\mathbf{d}}$, appear under the column entitled "Sample relative frequency" in the row entitled "Average." Thus, $P S(\overline{\mathrm{d}}, \mathrm{d})=0.480[$ see Eq. (5)]. The values of the reliability and (new) resolution terms for this collection of forecasts are 0.136 and 0.330 , respectively. Thus, from (6), $P S(\mathbf{r}, \mathbf{d})=0.286(0.480+0.136-0.330) \quad$ (cf., Murphy, 1972a, Table 3). The value of the original

\begin{tabular}{|c|c|c|c|c|c|}
\hline $\begin{array}{c}\text { Subcollection } \\
\text { number } \\
t\end{array}$ & $\begin{array}{l}\text { Forecast } \\
\mathbf{r}^{t}\end{array}$ & $\begin{array}{c}\text { Number } \\
\text { of } \\
\text { forecasts } \\
K^{t}\end{array}$ & $\begin{array}{c}\text { Sample } \\
\text { relative } \\
\text { frequency } \\
\overline{\mathbf{d}}^{t}\end{array}$ & $\begin{array}{c}\text { Subcollection } \\
\text { reliability } \\
K^{t}\left(\mathbf{r}^{t}-\overline{\mathbf{d}}^{t}\right)\left(\mathbf{r}^{t}-\overline{\mathbf{d}}^{t}\right)^{\prime}\end{array}$ & $\begin{array}{c}\text { Subcollection } \\
\text { resolution } \\
K^{t}(\overline{\mathbf{d}} t-\overline{\mathbf{d}})(\overline{\mathbf{d}}:-\overline{\mathbf{d}})^{\prime}\end{array}$ \\
\hline $\begin{array}{l}1 \\
2 \\
3 \\
4 \\
5 \\
6 \\
7\end{array}$ & $\begin{array}{l}(0.1,0.9) \\
(0.2,0.8) \\
(0.4,0.6) \\
(0.6,0.4) \\
(0.7,0.3) \\
(0.8,0.2) \\
(0.9,0.1)\end{array}$ & $\begin{array}{l}1 \\
4 \\
1 \\
1 \\
1 \\
1 \\
1\end{array}$ & $\begin{array}{l}(0.00,1.00) \\
(0.25,0.75) \\
(1.00,0.00) \\
(1.00,0.00) \\
(1.00,0.00) \\
(1.00,0.00) \\
(1.00,0.00)\end{array}$ & $\begin{array}{l}0.02 \\
0.02 \\
0.72 \\
0.32 \\
0.18 \\
0.08 \\
0.02\end{array}$ & $\begin{array}{l}0.72 \\
0.98 \\
0.32 \\
0.32 \\
0.32 \\
0.32 \\
0.32\end{array}$ \\
\hline Total & & 10 & & 1.36 & 3.30 \\
\hline Average & & & $(0.60,0.40)$ & 0.136 & 0.330 \\
\hline
\end{tabular}

TABLE 2. The new vector partition of the PS for the sample collection of forecasts presented in Table 1. 
TABLE 3. A sample collection of forecasts and the relevant observations in the three-state situation.

\begin{tabular}{ccc}
\hline $\begin{array}{c}\text { Forecast } \\
\text { Observation } \\
\text { number } \\
k\end{array}$ & $\begin{array}{c}\text { Forecast } \\
\mathbf{r}_{k}\end{array}$ & $\begin{array}{c}\text { Observation } \\
\mathbf{d}_{k}\end{array}$ \\
\hline 1 & $(0.1,0.3,0.6)$ & $(0,0,1)$ \\
2 & $(0.1,0.7,0.2)$ & $(0,1,0)$ \\
3 & $(0.3,0.5,0.2)$ & $(0,1,0)$ \\
4 & $(0.5,0.4,0.1)$ & $(0,1,0)$ \\
5 & $(0.7,0.3,0.0)$ & $(1,0,0)$ \\
6 & $(0.6,0.1,0.3)$ & $(0,0,1)$ \\
7 & $(0.5,0.4,0.1)$ & $(1,0,0)$ \\
8 & $(0.1,0.8,0.1)$ & $(0,1,0)$ \\
9 & $(0.1,0.6,0.3)$ & $(0,0,1)$ \\
10 & $(0.1,0.7,0.2)$ & $(0,0,1)$ \\
\hline
\end{tabular}

resolution term for this collection of forecasts is 0.150 (Murphy, 1972a, Table 3). Note that, as indicated in (7), $P S(\overrightarrow{\mathbf{d}}, \mathbf{d})$ is equal to the sum of the values of the two resolution terms (i.e., $0.480=0.330+0.150$ ).

A sample collection of ten $(K=10)$ forecasts in the three-state situation is presented in Table 3 (cf. Murphy, 1972b, Table 1b). The new vector partition of the PS for these forecasts is presented in Table 4. The sample relative frequencies for this collection of forecasts, $\overline{\mathrm{d}}$, appear in the same location in Table 4 as that in which they appeared in Table 2 for the collection of forecasts in the two-state situation. Thus, $P S(\overrightarrow{\mathrm{d}}, \mathbf{d})$ $=0.640$ [see Eq. (5)]. The values of the reliability and resolution terms for this collection of forecasts are 0.292 and 0.440 , respectively. Thus, from (6), $P S(\mathbf{r}, \mathbf{d})$ $=0.492(0.640+0.292-0.440)$ (cf., Murphy, 1972b, Table 3). The value of the original resolution term for this collection of forecasts is 0.200 (Murphy, 1972b, Table 3). As in the two-state situation, $P S(\overline{\mathbf{d}}, \mathbf{d})$ is equal to the sum of the values of the two resolution terms (i.e., $0.640=0.440+0.200$ ).

\section{The new and original partitions : A comparison}

In this section we briefly compare the new and original vector partitions of the $P S$. In particular, we consider the nature of the attributes of the forecasts with which the respective partitions are concerned, the interpretation of the partitions in geometric terms, and the use of the partitions as the bases for the formulation of measures to evaluate probability forecasts.

With regard to the attributes of the forecasts with which these partitions are concerned, recall that the terms in the new partition measure the uncertainty, reliability and resolution of the forecasts, and that the terms in the original partition measure the reliability and resolution of the forecasts. In addition, recall that the term resolution refers to different attributes of the forecasts in the two partitions. Since both partitions are concerned with reliability and only the new partition contains a measure of uncertainty, we are primarily concerned with the nature of the two resolution terms. As indicated in Section 3, the new resolution term measures the degree to which the sample relative frequencies for the $T$ subcollections of forecasts differ from the sample relative frequencies for the entire collection of forecasts. That is, this term is a measure of the ability of the forecaster to separate the occasions of concern into subcollections for which the sample relative frequencies differ from the sample relative frequencies for the collection of forecasts. On the other hand, as indicated in Section 2, the original resolution term measures the degree to which the sample relative frequencies for the $T$ subcollections differ from either the null or unity vectors. Thus, this term is a measure of the ability of the forecaster to separate the occasions of concern into subcollections for which the semple relative frequencies approach one of these two vectors. Therefore, this term is similar, in certain respects, to the measure of sharpness $S(\mathbf{r})$, where

$$
S(\mathbf{r})=(1 / K) \sum_{t=1}^{T} K^{t} \mathbf{r}^{t}\left(\mathbf{u}-\mathbf{r}^{t}\right)^{\prime}
$$

except that this measure is concerned with forecasts while the (original) measure of resolution is concerned with observations. In this regard, however, the term

TABLE 4. The new vector partition of the PS for the sample collection of forecasts presented in Table 3.

\begin{tabular}{|c|c|c|c|c|c|}
\hline $\begin{array}{c}\text { Subcollection } \\
\text { number } \\
t\end{array}$ & $\begin{array}{l}\text { Forecast } \\
\mathbf{r}^{t}\end{array}$ & $\begin{array}{l}\text { Number } \\
\text { of } \\
\text { forecasts } \\
K^{t}\end{array}$ & $\begin{array}{c}\text { Sample } \\
\text { relative } \\
\text { frequency } \\
\overline{\mathbf{d}}^{t}\end{array}$ & $\begin{array}{c}\text { Subcollection } \\
\text { reliability } \\
K^{t}\left(\mathbf{r}^{t}-\overline{\mathbf{d}}^{t}\right)\left(\mathbf{r}^{t}-\overline{\mathbf{d}}^{t}\right)^{\prime}\end{array}$ & $\begin{array}{c}\text { Subcollection } \\
\text { resolution } \\
K^{t}\left(\overline{\mathbf{d}^{t}}-\overline{\mathbf{d}}\right)\left(\overline{\mathbf{d}}^{t}-\overline{\mathbf{d}}\right)^{\prime}\end{array}$ \\
\hline $\begin{array}{l}1 \\
2 \\
3 \\
4 \\
5 \\
6 \\
7 \\
8\end{array}$ & $\begin{array}{l}(0.1,0.3,0.6) \\
(0.1,0.6,0.3) \\
(0.1,0.7,0.2) \\
(0.1,0.8,0.1) \\
(0.3,0.5,0.2) \\
(0.5,0.4,0.1) \\
(0.6,0.1,0.3) \\
(0.7,0.3,0.0)\end{array}$ & $\begin{array}{l}1 \\
1 \\
2 \\
1 \\
1 \\
2 \\
1 \\
1\end{array}$ & $\begin{array}{l}(0.0,0.0,1.0) \\
(0.0,0.0,1.0) \\
(0.0,0.5,0.5) \\
(0.0,1.0,0.0) \\
(0.0,1.0,0.0) \\
(0.5,0.5,0.0) \\
(0.0,0.0,1.0) \\
(1.0,0.0,0.0)\end{array}$ & $\begin{array}{l}0.26 \\
0.86 \\
0.28 \\
0.06 \\
0.38 \\
0.04 \\
0.86 \\
0.18\end{array}$ & $\begin{array}{l}0.56 \\
0.56 \\
0.12 \\
0.56 \\
0.56 \\
0.52 \\
0.56 \\
0.96\end{array}$ \\
\hline Total & & 10 & & 2.92 & 4.40 \\
\hline Average & & & $(0.2,0.4,0.4)$ & 0.292 & 0.440 \\
\hline
\end{tabular}




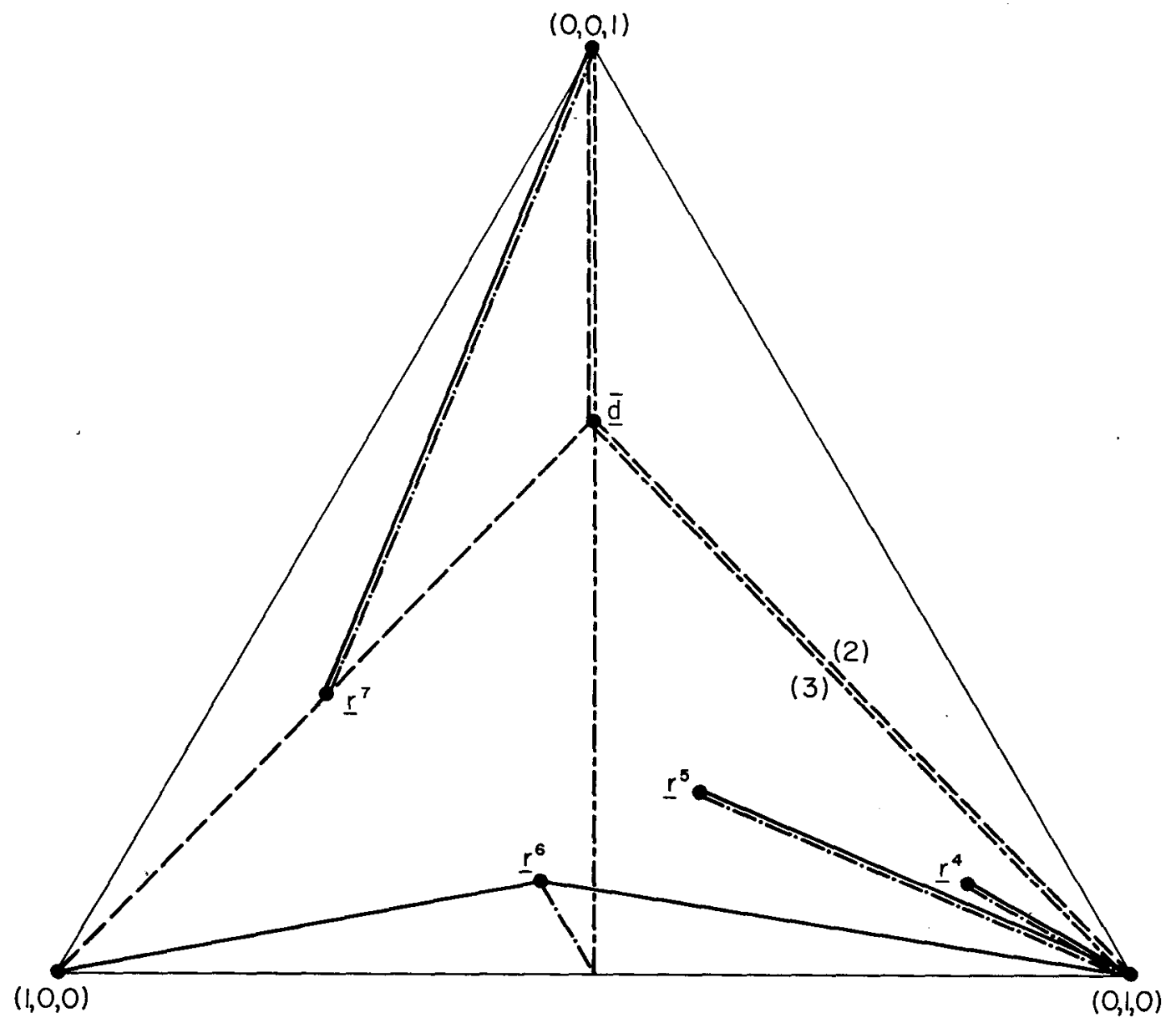

FIG. 1. A representation of the terms in the new vector partition of the $P S$ within the geometrical framework described in the text for a subset of the collection of ten forecasts presented in Table 3 ; specifically, for the five forecasts, $\mathbf{r}^{t}$, in the four subcollections for which $t=4,5,6$ and 7 . Contributions to the terms $\bar{D}^{2}(\mathbf{r}, \mathbf{d}), \overline{D^{2}}(\overline{\mathbf{d}}, \mathbf{d})$, $\widetilde{D^{2}}\left(\mathbf{r}^{t}, \overline{\mathbf{d}}^{t}\right)$, and $\overline{D^{2}}(\overline{\mathbf{d}} t, \overrightarrow{\mathbf{d}})$ are represented by solid, dashed, dashed-dotted, and long-and-short dashed lines, respectively. The numbers in parentheses adjacent to certain lines indicate the number of times that this particular distance occurs for this subset of forecasts (the absence of a number indicates that the distance occurs only once).

resolution appears to provide a better description of the attribute of concern in the new partition than that of concern in the original partition. In addition, we believe that, from the point of view of the evaluation of probability forecasts, the attribute of concern and the measure of resolution in the new partition are more meaningful than the attribute of concern and the measure of resolution in the original partition.

Murphy $(1972 a, b)$ has shown that vector forecasts and observations can be represented geometrically within the framework of a regular, $(N-1)$-dimensional simplex. The (Euclidean) distance between two points $\mathbf{r}$ and $\mathbf{r}^{*}$ in this framework is $D\left(\mathbf{r}, \mathbf{r}^{*}\right)$, where

$$
D\left(\mathbf{r}, \mathbf{r}^{*}\right)=\left[\left(\mathbf{r}-\mathbf{r}^{*}\right)\left(\mathbf{r}-\mathbf{r}^{*}\right)^{\prime}\right]^{\frac{1}{3}}
$$

(see Murphy, 1972c). Therefore, the new vector partition of the $P S, P S(\mathbf{r}, \mathbf{d})$, in (6), can be expressed as

$$
\overline{D^{2}}(\mathbf{r}, \mathbf{d})=\overline{D^{2}}(\overline{\mathbf{d}}, \mathbf{d})+\overline{D^{2}}\left(\mathbf{r}^{t}, \overline{\mathbf{d}}^{t}\right)-\overline{D^{2}}\left(\overline{\mathbf{d}}^{i}, \mathbf{d}\right),
$$

where $\overline{D^{2}}(\mathbf{r}, \mathbf{d})$ is the average of the squared distances between the $K$ forecasts $\mathbf{r}_{k}$ and the $K$ relevant observations $\mathbf{d}_{k}(k=1, \ldots, K) ; \overline{D^{2}}(\overline{\mathbf{d}}, \mathbf{d})$ is the average of the squared distances between the sample relative frequencies for the collection of forecasts, $\overline{\mathrm{d}}$, and the $K$ observations $\mathbf{d}_{k}(k=1, \ldots, K) ; \overline{D^{2}}\left(\mathbf{r}^{t}, \overline{\mathbf{d}}^{t}\right)$ is the weighted average of the squared distances between the $T$ distinct forecasts $\mathbf{r}^{t}$ and the relevant sample relative frequencies $\overline{\mathrm{d}}^{t}(t=1, \ldots, T)$; and $\overrightarrow{D^{2}}\left(\overline{\mathrm{d}}^{t}, \overrightarrow{\mathrm{d}}\right)$ is the weighted average of the squared distances between the sample relative frequencies for the $T$ subcollections of forecasts, $\overline{\mathrm{d}}^{t}$, and the sample relative frequencies for the collection of forecasts, $\overline{\mathrm{d}}(t=1, \ldots, T)$. Thus, all of the terms in the new partition of the $P S$ can be represented within this geometrical framework. The new partition of the $P S$ is depicted within this framework; an equilateral triangle in the three-state $(N=3)$ situation, in Fig. 1 for five of the ten forecasts presented in Table 3. The 
legend contains a detailed description of the contents of this figure. While the terms in the new vector partition can be depicted within this framework, the resolution term in the original partition, and, as a result, the original vector partition itself, cannot be direclly depicted within this framework.

The need for appropriate measures to evaluate probability forecasts has recently been indicated by Julian and Murphy (1972). In particular, Hughes (1971) has emphasized the need for measures to evaluate the "skill" of probability forecasts in order to be able to compare forecasters, forecast offices, etc. In this regard, note that the new vector partition of the $P S$ contains a term that represents the $P S$ for the sample relative frequencies, $P S(\overline{\mathbf{d}}, \mathbf{d})$, and that the latter can be considered to be "estimates" of the climatological probabilities. In a forthcoming paper Murphy (1973) describes a sample "skill score" which is formulated in terms of this partition and indicates that this skill score appears to offer certain advantages vis-à-vis than other skill scores proposed heretofore.

The new vector partition of the $P S$, then, appears to be more appropriate than the original vector partition of the $P S$.

\section{Summary and conclusion}

In this paper we have described a new vector partition of the probability, or Brier, score $(P S)$. This partition consists of three terms: 1) a measure of the uncertainty inherent in the events, or states, on the occasions of concern; 2) a measure of the reliability of the forecasts; and 3) a new measure of the resolution of the forecasts. This partition is compared with the original vector partition of the $P S$ and the relationship between the terms in these partitions is indicated. Two collections of forecasts are used to illustrate the differences between these partitions. Finally, the two partitions are compared with particular reference to the attributes of the forecasts with which they are concerned, their interpretation in geometric terms, and their use as the bases for the formulation of measures to evaluate probability forecasts. The results of these conparisons indicate that the new vector partition offers certain advantages vis-à-vis the original vector partition.

We could also formulate a new scalar partition of the $P S$ in which the forecasts of concern are individual probabilities rather than sets of probabilities. This new scalar partition would have the same relationship to the original scalar partition of the $P S$ (see Murphy, $1972 \mathrm{a}, \mathrm{b}$ ) as the new vector partition has to the original vector partition. We have restricted our attention to vector partitions of the $P S$ in this paper because vector partitions are, in general, more appropriate than scalar partitions (Murphy, 1972a, b).

\section{REFERENCES}

Brier; G. W., 1950: Verification of forecasts expressed in terms of probability. Mon. Wea. Rev., 78, 1-3.

Hughes, L. A., 1971: Comments on "Nonlinear utility and the probability score." J. Appl. Meteor., 10, 335 .

Julian, P. R., and A. H. Murphy, 1972: Probability and statistics in meteorology: A review of some recent developments. Bull. Amer. Meteor. Soc., 53, 957-965.

Murphy, A. H., 1972a: Scalar and vector partitions of the probability score: Part I. Two-state situation. J. A ppl. Meteor., 11, 273-282.

-_- 1972b: Scalar and vector partitions of the probability score: Part II. N-state situation. J. Appl. Meteor., 11, 1183-1192. 1972c: Ordinal relationships between measures of the "accuracy" and "value" of probability forecasts : Preliminary results. Tellus, 24, 531-542.

___ 1973: A sample skill score for probability forecasts. Boulder, Colo., National Center for Atmospheric Research, Advanced Study Program, unpubl. ms.

Sanders, F., 1958: The evaluation of subjective probability forecasts.. Cambridge, Massachusetts Institute of Technology, Dept. of Meteor., Sci. Rept. No. 5 [Contract AF 19(604)$1305], 62 \mathrm{pp}$.

, 1963: On subjective probability forecasting. $J . A p p l$. Meteor., 2, 191-201. 